\title{
Molecular modelling of membrane activity of amphotericin B, a polyene macrolide antifungal antibiotic ${ }^{\star}$
}

\author{
Maciej Baginski ${ }^{\bowtie}$, Kamil Sternal, Jacek Czub and Edward Borowski \\ Department of Pharmaceutical Technology and Biochemistry, Faculty of Chemistry, Gdansk University of Tech- \\ nology, Gdańsk, Poland; ${ }^{\circledR}$-mail: maciekb@hypnos.chem.pg.gda.pl
}

Received: 15 March, 2005; revised: 06 June, 2005; accepted: 28 July, 2005

available on-line: 05 August, 2005

\begin{abstract}
Amphotericin B $(\mathrm{AmB})$ is a well known polyene macrolide antibiotic used to treat systemic fungal infections. Despite its toxicity $\mathrm{AmB}$ is still regarded as a life-saving drug. The lack of adequate knowledge of the AmB mechanism of action is a serious obstacle to efficient development of new less toxic derivatives. Complementary to various experimental approaches, computational chemistry methods were used to study AmB mechanism of action. A programme lasting for a decade, that was run by our group covered studies of: i) molecular properties of AmB and its membrane targets, ii) structure and properties of AmB membrane channels, and iii) interaction of AmB with the membrane.
\end{abstract}

Keywords: amphotericin B, cholesterol, ergosterol, antifungal agents, lipid membrane, molecular modelling

Amphotericin B (AmB) (Fig. 1) belongs to a group of polyene macrolide antibiotics used to treat systemic fungal infections (reviewed by Omura \& Tanaka, 1984). Because of a lack of better alternatives, AmB despite its toxicity (mainly nephrotoxicity) is still regarded as a life-saving drug in curing deep-seated fungal infections (reviewed by Gallis et al., 1990; Hartsel \& Bolard, 1996). Apart from its toxicity, AmB exhibits several positive features indispensable for an effective antifungal drug, i.e.: i) high antifungal activity, ii) broad antifungal spectrum, iii) fungicidal activity, and iv) very rare induction of fungal resistance as well as the ability to overcome multidrug resistance of fungi. No other antifungal drug exhibits all these positive chemotherapeutic features together. Therefore, AmB may still be regarded as a promising candidate or rather a lead compound for the development of less toxic drugs, a new generation of derivatives of the parent molecule (Borowski, 2000). However, studies on $\mathrm{AmB}$ mechanism of action revealed that the biological action of AmB is very complex (Gale, 1984; Bolard, 1986; Hartsel et al., 1993; Hartsel \& Bolard, 1996). The lack of adequate knowledge of this mechanism is a serious obstacle to efficient development of new derivatives on a rational basis. The cell membrane is the site of action of this antibiotic. AmB interacts with membrane components and forms trans-membrane channels that disturb the barrier function of the membrane. The channels are responsible for induced cation leakage that eventually leads to cell death. According to the most popular sterol hypothesis, sterol molecules are indispensable for $\mathrm{AmB}$ action in the membrane. It is also regarded that chemotherapeutic application of the antibiotic is based on the higher affinity/activity of AmB towards membranes containing ergosterol (in fungal membranes) than cholesterol (in mammalian membranes) (for reviews see: Kotler-Brajtburg et al., 1974; Gale, 1984; Kerridge, 1986). Unfortunately, extensive research has not led to the recognition of a detailed molecular mechanism of action of AmB in the membrane. Therefore, complementary to various experimental approaches, computational chemistry methods were introduced in the late eighties to study AmB mechanism of action. These molecular modelling/computational chemistry efforts were undertaken in several laboratories but the contribution of our group to the field brings new essential data. A programme lasting for a decade, that was run in our laboratory covered studies of: i) molecular properties of $\mathrm{AmB}$ and its membrane targets, ii)

^Presented at the International Review Conference on Biotechnology, Vienna, Austria, November 2004.

Abbreviations: AmB, amphotericin B; DMPC, 1,2-dimyristoyl-sn-glycero-3-phosphocholine; MD, molecular dynamics; MEP, molecular electrostatic potential. 
AmB-induced membrane channels, and iii) different modes of interaction of AmB with the membrane depending on the association state of the antibiotic and its concentration in the membrane. Currently the studies include also AmB derivatives which exhibit lower toxicity than the parent molecule. Obtained results may help to understand the AmB mechanism of action on the molecular level and eventually to design new less toxic AmB derivatives.

\section{MOLECULAR PROPERTIES OF AmB AND ITS MEMBRANE TARGETS}

Studies of the molecular properties concern $\mathrm{AmB}$ and both sterols, ergosterol and cholesterol (Baginski, 1995). Comparative conformational analysis of both sterols revealed that ergosterol due to the lower conformational freedom of the side chain may have better van der Waals contacts with an AmB molecule (Baginski et al., 1989). Conformational analysis of the AmB molecule on the other hand showed that the mutual position of the aminosugar moiety and the macrolide ring is restricted (Baginski et al., 1997a). It was found that only so called "open" and "closed" conformations defined by $\phi, \psi$ dihedral angles (Fig. 1) can be acquired by a molecule of AmB (Berges et al., 1990; Meddeb et al., 1992). The carboxyl and amino groups in the "open" conformation are ready to form intermolecular hydrogen bonds, while in the "closed" conformation they can form only intramolecular hydrogen bonds via water molecules. Studies of AmB amide derivatives revealed that the "open" conformation is more favourable than the "closed" one (Resat et al., 2000).

Molecular electrostatic potential (MEP) calculations performed for AmB, cholesterol, and ergosterol molecules in different environments revealed that ergosterol exhibits a very different electrostatic pattern compared to a cholesterol molecule (Baginski et al., 1994; Baginski \& Borowski, 1997). Especially the carbon-carbon double bond in the side chain of ergosterol generates negative potential that in some way may better fit the distribution of MEP for AmB. On the other hand, MEP calculations for AmB showed amphipathic character of this molecule (Baginski \& Borowski, 1997). Based on the MEP calculations a hypothesis of a binary AmB-sterol complex was introduced (Baran \& Mazerski, 2002). However, this complex may correspond only to a situation when both AmB and sterol molecules are present in water and not in lipid environment.

\section{AmB MEMBRANE CHANNELS}

Due to the development of computational chemistry methods it was possible to perform molecular dynamics calculations of AmB membrane channels embedded in the lipid DMPC environment (Baginski et al., 1997b; 2002). These studies were aimed at understanding what are the molecular properties of such channels. The hypothetical structures of such channels (Fig. 2) proposed in the seventies were used as models (De Kruijff \& Demel, 1974). These models contain eight $\mathrm{AmB}$ and eight sterol molecules. It was found that the carboxyl and amino groups of AmB molecules form a chain of intermolecular hydrogen bonds. These strong intermolecular AmB-AmB interactions within the channel are responsible for its stability. This finding is in agreement with the experimental data and supports the idea that modification of either the carboxyl or the amino group modifies the activity or selectivity of a particular AmB derivative. Analysis of molecular dynamics (MD) data also revealed substantial differences between AmB-ergosterol and AmB-cholesterol channels (Baginski et al., 2002). The former ones are wider and more stable because the chain of hydrogen bonds between AmB molecules in the channel is more efficient. This may explain why AmB channels are more effective in membranes containing ergosterol molecules.

Taking into account models of AmB channels it was possible to study permeability properties of these pores (Resat \& Baginski, 2002). Thermodynamics and Monte Carlo studies of the AmB channel permeability showed that both cations and anions can enter and pass through the pore. No substantial barriers for either cations or anions were found.

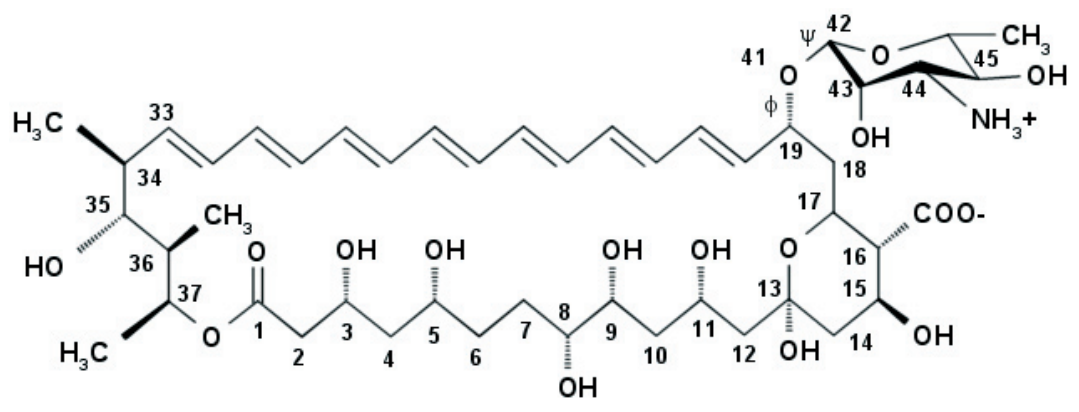

Figure 1. Structure of amphotericin B with partial numbering of heavy atoms and indication of $\phi, \psi$ dihedral angles. 


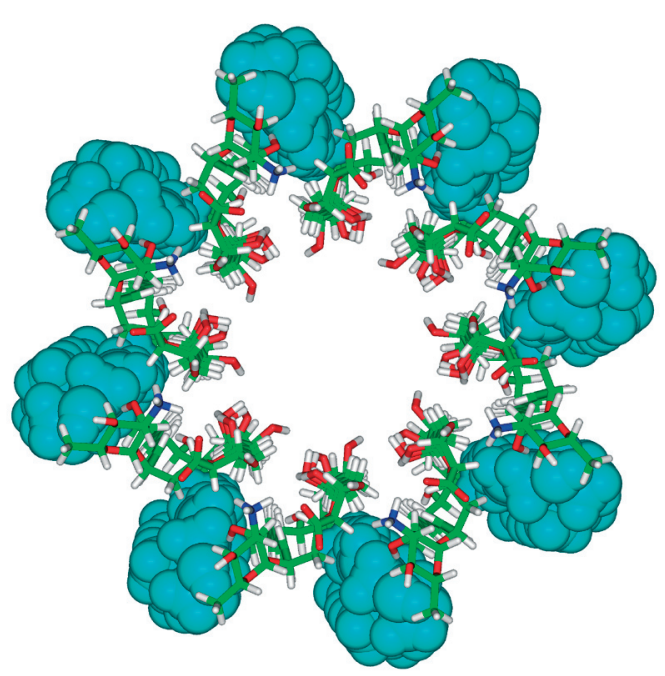

Figure 2. Model structure of AmB-ergosterol channel. The channel contains eight AmB molecules (sticks - coloured by atom type) and eight ergosterol molecules (cpk models - blue). The model comes from additional unpublished data from Baginski et al. (2002). It is a starting structure of the AmB channel model prepared for MD calculations generated by Insight II software (Accelrys, San Diego, USA).

This finding may be explained by the fact that AmB channels are quite large compared to many protein channels. It means that ions passing through the channel are not forced to strip out water molecules forming the solvation shell of each ion. On the other hand, differences between the diameters of AmB-ergosterol and AmB-cholesterol channels revealed that in the case of the AmB-cholesterol channel small potential well trap for cations can be formed at the entrance to the channel (our own data to be published). This observation may explain to some extent why AmB-cholesterol channels, even when formed, are not so good carriers of cations.

\section{INTERACTION OF AmB WITH THE MEMBRANE}

Not only the mechanism of AmB channel formation is unknown but also the mechanism of membrane entrance by $\mathrm{AmB}$ molecules is very unclear. One may suppose that monomers of AmB enter in some way the membrane, form binary complexes with lipid molecules and eventually form the channels. This mechanism may be called "sequential" one. On the other hand, one may imagine that supramolecular AmB complexes form at the membrane surface and subsequently enter the membrane undergoing further reorganisation towards functional channels. This may be called a "one step" mechanism.

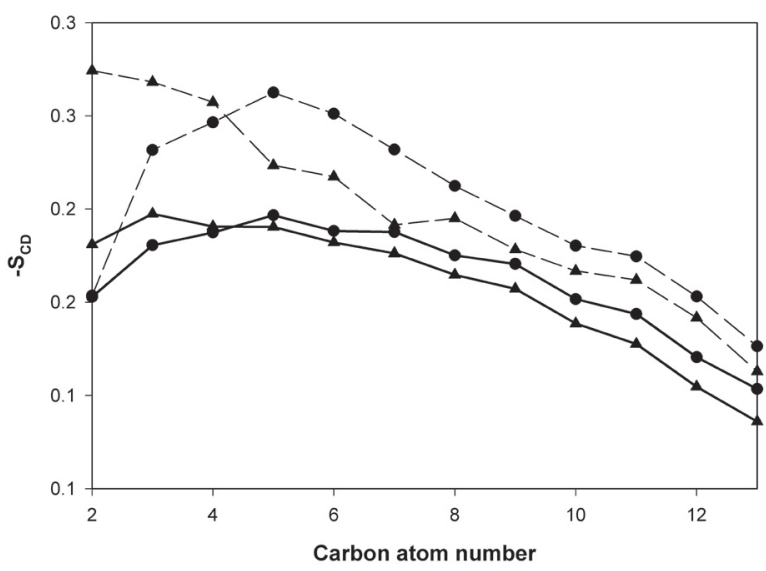

Figure 3. Order parameter of DMPC hydrocarbon chains (as a function of carbon atom position) for the lipid bilayer with AmB inserted perpendicularly into the membrane.

Dashed lines with triangles (sn1 chain) and dots (sn2 chain) describe averaged order parameters for five DMPC molecules close to AmB. Solid lines with triangles (sn1 chain) and dots (sn2 chain) describe averaged order parameters for other DMPC molecules. The order parameters were calculated, according to standard procedure, using molecular dynamic trajectories from a new project (manuscript in preparation) concerning interaction of $\mathrm{AmB}$ molecules with phospholipids inside the membrane.

To understand the way AmB molecules enter the membrane, molecular dynamics simulations were performed on DMPC membrane models with AmB molecules at the surface or inside the membrane (Sternal et al., 2004; Czub \& Baginski, 2004) ${ }^{1}$. The studies revealed that an AmB molecule can: i) either interact strongly in a horizontal position with the DMPC membrane surface or ii) tend to take vertical position with the polar head interacting with DMPC polar heads. The latter observation agrees with the experimental data showing ability of AmB molecules to form monolayers on air/water interface (Seoane et al., 1997). The position of the AmB molecule inside the membrane, on the other hand, is always vertical. It was found that $\mathrm{AmB}$ molecules strongly influence the membrane environment. In particular, it was found that an AmB molecule orders the surrounding acyl chains of DMPC molecules (Fig. 3). This observation together with previous experimental data may indicate that not only $\mathrm{AmB}-\mathrm{AmB}$ or $\mathrm{AmB}-$ sterol interactions are responsible for channel formation and $\mathrm{AmB}$ chemotherapeutic action.

\section{CONLUSIONS}

Molecular dynamics studies of AmB membrane channels revealed that free carboxyl and ami- 
no groups form a chain of intermolecular hydrogen bonds within the channel. These two groups are main targets for chemical AmB modifications and consequently their interactions within the channel may be influenced in order to get a more selective drug. It was also found that the structure of channels built from AmB and ergosterol is substantially different from that of channels built from $A m B$ and cholesterol. Analysis of interaction between AmB and the membrane surface shows specific orientation of AmB at the surface. The latter results indicate that a single AmB molecule is not prone to enter the membrane built only from phospholipid molecules. One may suppose that rather supramolecular structures have to be formed first on the membrane surface in order to enter the phospholipid membrane. However, the AmB molecule might enter membranes containing sterols in addition to phospholipids by a different way and this mechanism should be further studied by molecular modelling methods.

Altogether, the contribution made by molecular modelling studies gave new insight into the understanding of the molecular aspect of AmB mechanism of action. It may also give new impact on designing new, less toxic derivatives. At the same time the studies of AmB membrane interactions show that further work with more elaborate models containing also sterols in the membranes should be considered. Such studies are currently undertaken in our group.

\section{Acknowledgements}

The work was supported in part by grant 3 P05F 01225 from the State Committee for Scientific Research (KBN, Poland) and in part by internal grants from Gdansk University of Technology (Poland). The authors also thank the TASK Computational Centre (Gdansk) for CPU time.

\section{REFERENCES}

Baginski M (1995) Studies of selected molecular properties of amphotericin B and its cellular targets by theoretical chemistry methods. PhD Thesis. Technical University of Gdansk, Gdansk, Poland.

Baginski M, Borowski E (1997) Distribution of electrostatic potential around amphotericin B and its membrane targets. Theochem J Mol Struct 389: 139-146.

Baginski M, Tempczyk A, Borowski E (1989) Comparative conformational analysis of cholesterol and ergosterol by molecular mechanics. Eur Biophys J 17: 159-166.

Baginski M, Bruni P, Borowski E (1994) Comparative analysis of the distribution of the molecular electrostatic potential for cholesterol and ergosterol. Theochem J Mol Struct 311: 285-296.

Baginski M, Gariboldi P, Bruni P, Borowski E (1997a) Conformational analysis of amphotericin B. Biophys Chem 65: 91-100.
Baginski M, Resat H, McCammon JA (1997b) Molecular properties of amphotericin B membrane channel: A molecular dynamics simulation. Mol Pharmacol 52: 560-570.

Baginski M, Resat H, Borowski E (2002) Comparative molecular dynamics simulations of amphotericin B-cholesterol/ergosterol membrane channels. Biochim Biophys Acta 1567: 63-78.

Baran M, Mazerski J (2002) Molecular modelling of amphotericin B-ergosterol primary complex in water. Biophys Chem 95: 125-133.

Berges J, Caillet J, Langlet J, Gresh N, Herve M, Gary-Bobo CM (1990) Conformational study of the polar head of the amphotericin B in the isolated state and in the presence of water molecules. Stud Phys Theoret Chem 71: 253-263.

Bolard J (1986) How do the polyene macrolide antibiotics affect the cellular membrane properties? Biochim Biophys Acta 864: 257-304.

Borowski E (2000) Novel approaches in the rational design of antifungal agents of low toxicity. Il Farmaco 55: 206-208.

De Kruijff B, Demel RA (1974) Polyene antibiotic-sterol interactions in membranes of acholeplasma laidlawii cells and lecithin liposomes. III. Molecular structure of the polyene antibiotic-cholesterol complexes. Biochim Biophys Acta 339: 57-70.

Gale EF (1984) Mode of action and resistance mechanisms of polyene macrolides. In Macrolide Antibiotics. Omura S, eds, pp 425-455. Academic Press, Inc., Orlando.

Gallis HA, Drew RH, Pickard WW (1990) Amphotericin B: 30 years of clinical experience. Rev Infect Dis 12: 308 329.

Hartsel S, Bolard J (1996) Amphotericin B: new life for an old drug. Trends Pharmacol Sci 17: 445-449.

Hartsel SC, Hatch C, Ayenew W (1993) How does amphotericin B work?: Studies on model membrane systems. J Liposome Res 3: 377-408.

Kerridge D (1986) Mode of action of clinically important antifungal drugs. Adv Microb Physiol 27: 1-72.

Kotler-Brajtburg J, Price HD, Medoff G, Schlessinger D, Kobayashi GS (1974) Molecular basis for the selective toxicity of amphotericin B for yeast and filipin for animal cells. Antimicrob Agents Chemother 5: 377-382.

Meddeb S, Berges J, Caillet J, Langlet J (1992) Comparative theoretical study of the conformations of amphotericin methyl ester and amphotericin-B polar heads in the presence of water. Biochim Biophys Acta 1112: 266-272.

Omura S, Tanaka H (1984) Production, structure, antifungal activity of polyene macrolides. In Macrolide Antibiotics. Omura S, eds, pp 351-404. Academic Press, Inc., Orlando.

Resat H, Baginski M (2002) Ion passage pathways and thermodynamics of the amphotericin B membrane channel. Eur Biophys J Biophys Lett 31: 294-305.

Resat H, Sungur FA, Baginski M, Borowski E, Aviyente V (2000) Conformational properties of amphotericin B amide derivatives - impact on selective toxicity. J Comput Aided Mol Des 14: 689-703.

Seoane JR, Vila Romeu N, Minones J, Conde O, Dynarowicz-Latka P, Casas M (1997) The behaviour of amphotericin B monolayers at the air/water interface. Progr Colloid Polym Sci 105: 173-179.

Sternal K, Czub J, Baginski M (2004) Molecular aspects of the interaction between amphotericin $\mathrm{B}$ and a phospholipid bilayer: molecular dynamics studies. $\mathrm{J} \mathrm{Mol}$ Model 10: 223-232. 